Pacific Journal of Mathematics

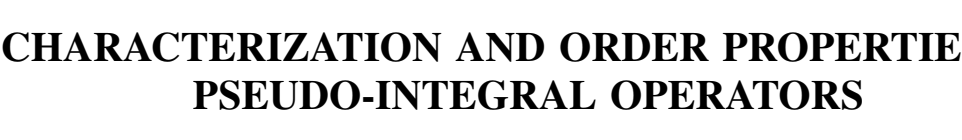




\title{
CHARACTERIZATION AND ORDER PROPERTIES OF PSEUDO-INTEGRAL OPERATORS
}

\author{
A. R. SOUROUR
}

Let $\left(X, \mathscr{A}, m_{1}\right)$ and $\left(Y, \mathscr{B}, m_{2}\right)$ be separable $\sigma$-finite measure spaces. A linear transformation $T$ from an order-ideal $L$ of measurable functions on $Y$ into the space of measurable functions on $X$ is called a pseudo-integral operator if it is induced by a measure $\mu$ on $X \times Y$ via the equation

$$
\int(T f)(x) g(x) m_{1}(d x)=\iint f(y) g(x) \mu(d x, d y)
$$

for sufficiently many functions $g$. Our main theorem states that $T$ is a pseudo-integral operator if and only if $T f_{n} \rightarrow 0$ a.e. whenever $0 \leqq f_{n} \leqq f \in L$ and $f_{n} \rightarrow 0$ a.e. We also study the order structure of the class of pseudo-integral operators showing that they form a band (order-closed ideal) in the space of order-bounded operators.

Introduction. Let $\left(X, \mathscr{A}, m_{1}\right)$ and $\left(Y, \mathscr{B}, m_{2}\right)$ be separable $\sigma$ finite measure spaces, and let $M(X)$ and $M(Y)$ be the linear spaces of equivalence classes of (real or complex) measurable functions on $X$ and $Y$ respectively. A linear operator $T$ from a linear subspace $L$ of $M(Y)$ into $M(X)$ is called an integral operator if there exists a measurable function $k$ on $X \times Y$ (called the kernel of $T$ ) such that for every $f$ in $L, T f$ is given by the equation $(T f)(x)=$ $\int f(y) k(x, y) m_{2}(d y)$ for $m_{1}$-almost every $x$. Arveson [2] introduced a more general class of operators, which he called pseudo-integral operators, associated with measures, rather than functions, on $X \times Y$. By a pseudo-integral operator we mean an operator given by the equation

$$
\int(T f)(x) g(x) m_{1}(d x)=\iint f(y) g(x) \mu(d x, d y),
$$

for sufficiently many functions $g$ (this will be made precise later). If $(Y, \mathscr{B})$ is a standard Borel space, then $T$ can be given explicitely by the equation

$$
(T f)(x)=\int f(y) \mu_{x}(d y)
$$

where $\left\{\mu_{x}\right\}$ is a certain family of measures on $Y$, related to $\mu$ via the theorem on disintegration of measures.

The purpose of this paper is to give a necessary and sufficient condition for an operator between spaces of measurable functions to 
be a pseudo-integral operator. We also study the order structure of the class of pseudo-integral operators and show that they form a band in the vector lattice of order-bounded operators.

The "natural domain" of an integral or a pseudo-integral operator is an order-ideal of $M(Y)$, that is, a linear subspace $E$ of $M(Y)$ such that $f \in E$ whenever $|f| \leqq g \in E$. In view of this, we will consider operators from an order-ideal $L$ of $M(Y)$ into $M(X)$. Our main result (Theorem 5.2) states that $T$ is a pseudo-integral operator if and only if it satisfies the condition: whenever $\left|f_{n}\right| \leqq f \in L$ and $f_{n} \rightarrow 0$ almost everywhere, we must have $T f_{n} \rightarrow 0$ almost everywhere. A similar characterization of integral operators has been obtained by $\mathrm{A}$. V. Bukhvalov [6].

TheORem (Bukhvalov). A linear operator $T$ from $L$ into $M(X)$ is an integral operator if and only if it satisfies the condition: $T f_{n} \rightarrow 0$ almost everywhere whenever $\left|f_{n}\right| \leqq f \in L$ and $f_{n} \rightarrow 0$ in measure on every subset of $Y$ of finite measure.

Our approach is different from Bukhvalov's.

1. Preliminaries. For clarity of exposition we will deal first with standard measure spaces. The generalizations to general separable measure spaces will be indicated toward the end of the paper. Let $\left(X, \mathscr{A}, m_{1}\right)$ be an arbitrary measure space and $\left(Y, \mathscr{B}, m_{2}\right)$ a standard Borel space, i.e., $(Y, \mathscr{B})$ is Borel-isomorphic to a Borel subset of a complete separable metric space (see [13] or [3, Chapter 3]). The measures $m_{1}$ and $m_{2}$ are positive and finite. (Our results are valid for $\sigma$-finite measures and follow immediately from the finite case.) Let $L$ be an order-ideal in $M(Y)$, and we will assume that $1 \in L$. This assumption is not essential (see Remark 2.7), but will simplify statements and proofs.

All linear spaces are over the real or the complex numbers. The proofs will be carried out only for the real case. As usual, equality between two members of $M(X)$ or $M(Y)$ will mean equivalence module sets of measure zero. The measure being $m_{1}$ or $m_{2}$. The characteristic function of a set $E$ will be denoted by $1_{E}$

Definition 1.1. A kernel is a map $x \rightarrow \mu_{x}$ of $X$ into the space of bounded Borel measures on $Y$ satisfying the following two conditions.

(i) If $B \in \mathscr{B}$ and $m_{2}(B)=0$, then $\mu_{x}(B)=0$ for $m_{1}$-almost every $x$.

(ii) For every $B$ in $\mathscr{B}$, the maps $x \rightarrow \mu_{x}(B)$ and $x \rightarrow\left|\mu_{x}\right|(B)$ are Borel function. 
The domain of the kernel $x \rightarrow \mu_{x}$ is the ideal

$$
L_{\mu}=\left\{f: f \in M(Y), f \in L^{1}\left(\left|\mu_{x}\right|\right) \text { for } m_{1} \text {-almost every } x\right\} .
$$

The kernel $x \rightarrow \mu_{x}$ will also be denoted by $\left\{\mu_{x}\right\}$.

Definition 1.2. A linear operator $T$ from an ideal $L \subset M(Y)$ into $M(X)$ is called a pseudo-integral operator if there is a kernel $\left\{\mu_{x}\right\}$ such that $L \subset L_{\mu}$ and

$$
(T f)(x)=\int f(y) \mu_{x}(d y) \text { a.e. } \quad\left(m_{1}\right)
$$

for every $f$ in $L$.

For examples and counterexamples of pseudo-integral operators on $L^{2}(Y)$, see [17].

REMARKS 1.3. (i) The kernel is uniquely determined by the operator in the sense that if $\left\{\mu_{x}\right\}$ and $\left\{\nu_{x}\right\}$ are kernels of the same operator, the $\mu_{x}=\nu_{x}$ for $m_{1}$-almost every $x$. This follows from the fact that $\mathscr{B}$ has a countable generating family $\left\{B_{n}\right\}$, (see [13]), and the observation that $\mu\left(x, B_{n}\right)=\left(T 1_{B_{n}}\right)(x)=\nu\left(x, B_{n}\right)$ for $m_{1}$-almost every $x$.

(ii) The domain of $\left\{\mu_{x}\right\}$ is the same as the domain of $\left\{\left|\mu_{x}\right|\right\}$. Consequently, if $T_{\mu}$ is an integral operator from $L$ into $M(X)$, then $\left\{\left|\mu_{x}\right|\right\}$ is the pseudo-kernel of an operator $T_{|\mu|}$ from $L$ into $M(X)$.

(iii) Let $\left\{\mu_{x}\right\}$ be a family of measures on $Y$ inducing an operator $T$ on $L$ by the equation $(T f)(x)=\int f(y) \mu_{x}(d y)$. Then $\left\{\mu_{x}\right\}$ obviously satisfy condition 1.1(i). Furthermore, by modifying the measures $\left\{\mu_{x}\right\}$ for $x$ in an $m_{1}$-null set, we obtain a kernel satisfying the measurability conditions 1.1(ii). This follows from our characterization theorem (Theorem 5.2), and we do not know of an independent proof. This problem is analogous to the question of measurability of the kernels of integral operators which was settled, in the affirmative, by Bukhvalov [6]. It is easy, however, to prove one half of 1.1 (ii), namely the measurability of the maps $x \rightarrow \mu_{x}(B)$ after modifying $\mu_{x}$ for $x$ in an $m_{1}$-null set if necessary. This follows from the equality $\mu_{x}(B)=\left(T 1_{B}\right)(x)$ a.e., and the fact that $\mathscr{B}$ is countably generated.

2. Measure kernels. We have defined a kernel as a map $x \rightarrow \mu_{x}$ of $X$ into the space of measures on $Y$. We wish to replace $\left\{\mu_{x}\right\}$ by one measure $\mu$ on the product space $(X \times Y, \mathscr{A} \otimes \mathscr{B})$. The measure $\mu$ is the product of $m_{1}$ and $\left\{\mu_{x}\right\}$. The only difficulty is the 
fact that $\int\left|\mu_{x}\right|(Y) m_{1}(d x)$ may be infinite. Therefore we must consider "measures" $\mu$ which are defined only on an ideal of sets $\mathscr{C}_{0}$ in $\mathscr{A} \otimes \mathscr{B}$. We will use the term local measure to refer to such an object, i.e., a countably additive complex-valued set function on an ideal of measurable sets. (This resembles a Radon measure except that we do not have any topology.) Equivalently, a local measure on a Borel space $(Z, \mathscr{C})$ is a product of a unimodular measurable function and a positive (extended real-valued) measure, that is $\mu(d z)=\phi(z)|\mu|(d z)$, where $|\phi(z)|=1$. A local measure $\mu$ is called $\sigma$-finite if $|\mu|$ is $\sigma$-finite. For $f \in L^{1}(|\mu|)$, define $\int f d \mu$ by $\int f(z) \mu(d z)=$ $\int f(z) \phi(z)|\mu|(d z)$.

Let $x \rightarrow \mu_{x}$ be a kernel, and let $X_{n}=\left\{x: n-1 \leqq\left|\mu_{x}\right|(Y)<n\right\}$. Thus $\left\{X_{n}\right\}$ are disjoint measurable sets and $X=\mathrm{U} X_{n}$. The product $\mu$ of $m_{1}$ and $\left\{\mu_{x}\right\}$ can be defined on the Borel subsets of $X_{n} \times Y$ by $\mu(d x, d y)=\mu_{x}(d y) m_{1}(d x)$, that is, $\mu(E)=\int_{X} \int_{Y} 1_{E}(x, y) \mu_{x}(d y) m_{1}(d x)$, for every Borel set $E$ in $X_{n} \times Y$. For the details of this construction, see [4, Theorem 2.6.2]. It is easy to see that $\mu$ extends to a local measure on $X \times Y$, and that $|\mu|(d x, d y)=\left|\mu_{x}\right|(d y) m_{1}(d x)$. It is also easy to see that $|\mu|$ vanishes on marginally null sets. (Recall that a measurable subsets of $X \times Y$ is called marginally null if it is a subset of a rectangle $A \times B$ with $m_{1}(A)=0$ or $m_{2}(B)=0$.)

The above construction is valid for any measure space. Under the assumption that $Y$ is a standard Borel space, we can recover $\left\{\mu_{x}\right\}$ from $\mu$ as shown below.

DEFINITION 2.1. By a measure kernel, we mean a local measure $\mu$ on $X \times Y$ satisfying the following two properties:

(i) There are countably many disjoint measurable sets $X_{n}$ such that $X=\bigcup X_{n}$, and $|\mu|\left(X_{n} \times Y\right)<\infty$ for every $n$.

(ii) $|\mu|$ vanishes on marginally null sets.

LEMMA 2.2. Let $\mu$ be a measure kernel. Then there exists a map $x \rightarrow \mu_{x}$ of $X$ into the set of all bounded Borel measures on $Y$ such that

(i) For $B$ in $\mathscr{B}$, the maps $x \rightarrow \mu_{x}(B)$ and $x \rightarrow\left|\mu_{x}\right|(B)$ are Borel functions, and are zero $\left(m_{1}\right.$-a.e. $)$ if $m_{2}(B)=0$.

(ii ) $\mu(d x, d y)=\mu_{x}(d y) m_{1}(d x)$.

(iii) $|\mu|(d x, d y)=\left|\mu_{x}\right|(d y) m_{1}(d x)$.

Moreover, the measures $\mu_{x}$ are essentially unique, i.e., if $\nu_{x}$ are measures satisfying (i) and (ii), then $\mu_{x}=\nu_{x}$ for $m_{1}$-almost every $x$.

Proof. Let $|\mu|_{1}$ be the first marginal of $|\mu|$, that is, $|\mu|_{1}(A)=$ 
$|\mu|(A \times Y)$. Condition 2.1(i) implies that $|\mu|_{1}$ is $\sigma$-finite, and condition 2.1(ii) implies that it is absolutely continuous with respect to $m_{1}$. The theorem on disintegration of measures ([1, Theorem 2] or [5, pp. 59-63] together with [2, p. 461]) gives a map $x \rightarrow p_{x}$ from $X$ into the space of probability measures on $Y$ such that:

(a) For every $B$ in $\mathscr{B}$, the map $x \rightarrow p_{x}(B)$ is a Borel function.

(b) $|\mu|(d x, d y)=p_{x}(d y)|\mu|_{1}(d x)$.

An examination of proofs in [1] and [5] shows that we need only require that $Y$ is standard.

Let $\lambda_{x}(d y)=k(x) p_{x}(d y)$, where $k$ is the Radon-Nikodym derivative of $|\mu|_{1}$ with respect to $m_{1}$. So $|\mu|(d x, d y)=\lambda_{x}(d y)_{m_{1}}(d x)$. Finally, let $\mu_{x}(d y)=\phi(x, y) \lambda_{x}(d y)$, where $\phi$ is the Radon-Nikodym derivative of $\mu$ with respect to $|\mu|$. Therefore $\mu(d x, d y)=\mu_{x}(d y) m_{1}(d x)$. It is also evident that $\left|\mu_{x}\right|=\lambda_{x}$.

To prove the uniqueness, let $\left\{\nu_{x}\right\}$ be another family of measures on $Y$ such that maps $x \rightarrow \nu_{x}(B)$ are Borel functions, and $\mu(d x, d y)=$ $\nu_{x}(d y) m_{1}(d x)$. Let $\left\{B_{n}\right\}$ be a countable generating family for $\mathscr{B}$. Then

$$
\int \nu_{x}\left(B_{n}\right) 1_{A}(x) m_{1}(d x)=\mu\left(A \times B_{n}\right)=\int \mu_{x}\left(B_{n}\right) 1_{A}(x) m_{1}(d x)
$$

whenever $A \in \mathscr{A},|\mu|(A \times Y)<\infty$. Thus $\mu_{x}\left(B_{n}\right)=\nu_{x}\left(B_{n}\right)$ for almost every $x$ and hence $\mu_{x}=\nu_{x}$ for almost every $x$. This ends the proof.

REMARK. It follows easily from conditions (ii) and (iii) of the Lemma that

$$
\mu^{+}(d x, d y)=\left(\mu_{x}\right)^{+}(d y) m_{1}(d x), \quad \text { and } \quad \mu^{-}(d x, d y)=\left(\mu_{x}\right)^{-}(d y) m_{2}(d x) .
$$

The term kernel will be used for both $\mu$ and $\left\{\mu_{x}\right\}$. When we wish to distinguish between the two, we will call $\mu$ the measure kernel and $\left\{\mu_{x}\right\}$ the disintegrated kernel.

Next we describe how the measure kernel $\mu$ directly induces an operator. For any $f$ in $M(Y)$, let $\mathscr{A}_{f, \mu}=\left\{A: A \in \mathscr{A}\right.$, and $f(y) 1_{A}(x) \in$ $\left.L^{1}(|\mu|)\right\}$ and $\mathscr{F}_{f, \mu}=\left\{g: g \in M(X)\right.$, and $\left.f(y) g(x) \in L^{1}(|\mu|)\right\}$. The domain $L_{\mu}$ of $\mu$ is defined to be the set of those functions $f$ in $M(Y)$ for which $\mathscr{A}_{f, \mu}$ generates $\mathscr{A}$ as a $\sigma$-algebra (equivalently, the ideal of functions $\mathscr{F}_{f, \mu}$ has support $X$ ). It is obvious that $L_{\mu}$ is an orderideal in $M(Y)$, and $1 \in L_{\mu}$.

LEMMA 2.3. Let $\mu$ be a measure kernel. Then $\mu$ induces an operator $T_{\mu}$ from $L_{\mu}$ into $M(X)$ by the equation

We also have

(i) $\int\left(T_{\mu} f\right)(x) 1_{A}(x) m_{1}(d x)=\iint f(y) 1_{A}(x) \mu(d x, d y) \quad$ for $\quad A \in \mathscr{A}_{f, \mu}$.

(ii) $\int\left(T_{\mu} f\right)(x) g(x) m_{1}(x d)=\iint f(y) g(x) \mu(d x, d y)$ for $g \in \mathscr{F}_{f, \mu}$. 
Proof. Let $f \in L_{k}$, and let $X=\bigcup X_{n}$ where $X_{n}$ are disjoint and $1_{x_{n}} \in \mathscr{C}_{f, u}$. Since it suffices to describe $T f$ on every $X_{n}$, we may assume that $1 \in \mathscr{A}_{f, \mu}$. For $A$ in $\mathscr{A}$, let $\lambda_{f}(A)=\iint f(y) 1_{A}(x) \mu(d x, d y)$. Thus $\lambda_{f}$ is a countably additive bounded measure on $X$, absolutely continuous with respect to $m_{1}$. Let $T_{\mu} f$ be the Radon-Nikodym derivative of $\lambda_{f}$ with respect to $m_{1}$. Then $T_{n}$ is the desired operator.

REMARK. The measure $\mu$ is uniquely determined by equation 2.3 (i) since it determines $\mu$ uniquely on enough rectangles to generate $\mathscr{A} \otimes \mathscr{B}$.

Observe that in the preceding Lemma, the condition that $Y$ is standard is not needed. In the case $Y$ is standard, we will show that the class of operators induced by measure kernels agrees with the class of pseudo-integral operators induced by disintegrated kernels.

Proposition 2.4. Let $\mu$ be measure kernel and $\left\{\mu_{x}\right\}$ its disintegration. Then the domain $L_{\mu}$ of $\mu$ is the domain of $\left\{\mu_{x}\right\}$. Furthermore the operator $T_{"}$ induced by $\mu$ (Lemma 2.3) agrees with operator induced by $\left\{\varkappa_{x}\right\}$ (Definition 1.2).

Proof. Apply the general Fubuini's theorem [4, Theorem 2.6.4].

COROLlary 2.5. The set of all pseudo-integral operators from a linear space.

Note that there is some difficulty in proving this directly from Definition 1.2. The difficulty lies in proving the measurability condition 1.1(ii) for the variation of the sum of two disintegrated kernels.

Proposition 2.6. Let $T$ be a pseudo-integral operator with measure kernel $\mu$ and let $\mu(d x, d y)=\mu_{x}(d y) m_{1}(d x)$. The following conditions are equivalent.

(i) $T$ is an integral operator.

(ii) $\mu$ is absolutely continuous with respect to $m_{1} \times m_{2}$.

(iii) $\mu_{x}$ is absolutely continuous with respect to $m_{2}$ for $m_{1}$-almost every $x$.

Proof. The implication (i) $\Rightarrow$ (iii) follows from the uniqueness of the kernel. The implication (ii) $\Rightarrow$ (i) is obvious. To prove that (iii) $\Rightarrow$ (ii) assume that $\mu_{x}(d y)=k(x, y) m_{2}(d y)$. The function $k$ is measurable in the second variable. (It may not be jointly measurable.) Let $E$ be a Borel subset of $X \times Y$ with $\left(m_{1} \times m_{2}\right)(E)=0$, and 
let $E^{x}=\{y:(x, y) \in E\}$. Thus $m_{2}\left(E^{x}\right)=0$ for $m_{1}$-almost every $x$, and hence $\mu_{x}\left(E^{x}\right)=0$ for $m_{1}$-almost every $x$. Thus $\mu(E)=$ $\int_{Y} \int_{X} \mu_{x}\left(E^{x}\right) m_{1}(d x)=0$, that is, $\mu$ is absolutely continuous.

REMARK 2.7. The condition that $1 \in L$ is not essential. Let $L$ be any order-ideal in $M(Y)$. By replacing $Y$ by the support of $L$, we may assume that the support of $L$ is all of $Y$ (i.e., $L$ is a foundation in the terminology of [18]). It is easy to see that there exists a disjoint countable family $\left\{Y_{n}\right\}$ of Borel subsets of $Y$ such that $Y=\bigcup Y_{n}$ and $1_{Y_{n}} \in L$ for every $n$. In the definition of a disintegrated kernel, the measures $\mu_{x}$ must be replaced by local measures which are uniformly $\sigma$-infinite. Similarly, in the definition of a measure kernel $\mu$, condition 2.1 (i) must be weakened to the following form: There is a countable disjoint family $\left\{Y_{n}\right\}$ of measurable subsets of $Y$, and for every $n$, a countable disjoint family $\left\{X_{n j}: j=\right.$ $1,2, \cdots\}$ of measurable subsets of $X$ such that $Y=\mathrm{U} Y_{n}, X=\mathrm{U}_{j} X_{n j}$ for every $n$, and $|\mu|\left(X_{n j} \times Y_{n}\right)<\infty$ for every $n$ and $j$. With these modifications, it is easy to see that all the results in this paper are still valid without the assumption that $1 \in L$.

3. Positive operators. In this section we prove the characterization theorem for positive operators, i.e., operators which map nonnegative functions into nonnegative functions.

As before we have finite measure spaces $\left(X, \mathscr{A}, m_{1}\right)$ and $\left(Y, \mathscr{B}, m_{2}\right)$ where $Y$ is standard. We also have an order-ideal $L$ of $M(Y)$ with $1 \in L$.

Lemma 3.1. A pseudo-integral operator is positive if and only if its measure kernel is positive.

Proof. The "if" part is trivial. To prove the "only if" part, let $T$ be a positive pseudo-integral operator with kernel $\mu$. Let $A \in \mathscr{A}, \quad B \in \mathscr{B}$ be such that $1_{A} T 1_{B} \in L^{1}\left(m_{1}\right)$, then $\mu(A \times B)=$ $\int 1_{A}(x)\left(T 1_{B}\right)(x) m_{1}(d x) \geqq 0$. There are enough such rectangles to generate $\mathscr{A} \otimes \mathscr{B}$, and so $\mu$ is a positive measure.

Note that $\mu$ is positive if and only if measures $\mu_{x}$ are positive for $m_{1}$-almost every $x$.

THEOREM 3.2. Let $T$ be a positive operator from $L$ into $M(X)$. The following conditions are equivalent.

(i) $T$ is a pseudo-integral operator.

(ii) $T$ is order-continuous, i.e., if $0 \leqq f_{n} \leqq f \in L$, and $f_{n} \rightarrow 0$ almost everywhere $\left(m_{2}\right)$, then $T f_{n} \rightarrow 0$ almost everywhere $\left(m_{1}\right)$. 
Proof. (i) $\Rightarrow$ (ii). Let $T$ be a pseudo-integral operator with kernel $\left\{\mu_{x}\right\}$, and let $0 \leqq f_{n} \leqq f \in L, f_{n} \rightarrow 0$ almost everywhere. Let $X_{j}=\{x:(T f)(x) \leqq j\}$. The dominated convergence theorem implies that $T f_{n} \rightarrow 0$ a.e. on every $X_{j}$. This proves (ii).

(ii) $\Rightarrow$ (i). Every standard Borel space is Borel-isomorphic to a compact metric space [13], so we may assume that $Y$ is a compact metric space and $\mathscr{B}$ is generated by the topology of $Y$. Let $C(Y)$ be the space of real-valued continuous functions on $Y$. Let $\left\{e_{n}: n=\right.$ $0,1,2, \cdots\}$ be a countable linearly independent subset of positive continuous functions such that $e_{0}=1$ and the linear span $\mathscr{D}$ of $\left\{e_{n}\right\}$ is dense in $C(Y)$. Let $\mathscr{D}_{r}$ be the linear manifold, over the rationals, spanned by $\left\{e_{n}\right\}$. For every $n$, Let $\pi\left\{T e_{n}\right\}$ be a function in the equivalence class $T e_{n}$. Extend $\pi$ by linearity to $T \mathscr{D}$. For every $x$, the map $\phi_{x}$, defined by $\phi_{x}(f)=\pi(T f)(x)$, is a linear map of $\mathscr{D}$ into the real numbers. There is an $m_{1}$-null set $X_{0}$ such that $\pi(T f)(x) \geqq 0$ for every $x \in X \backslash X_{0}$ and every nonnegative $f$ in $\mathscr{D}_{r}$.

We will show that $\phi_{x}$ is bounded on $\mathscr{D}$ for every $x$ in $X \backslash X_{0}$. First consider $f$ in $\mathscr{D}_{r}$ with $-1 \leqq f(y) \leqq 1$. By the positivity of $T$, we have $-h_{0}(x) \leqq \dot{\phi}_{x}(f) \leqq h_{0}(x)$, where $h_{0}=\pi(T 1)$. Thus $\phi_{x}$ is bounded on $\mathscr{D}_{r}$ with norm $h_{0}(x)$. For every positive integer $n$, let $\mathscr{M}_{n}$ be the linear span of $\left\{e_{1}, e_{2}, \cdots, e_{n}\right\}$. The norm of the map $\phi_{x} \mid \mathscr{A}_{n}$ is determined on the dense set $\mathscr{D}_{r} \cap \mathscr{M}_{n}$, so the norm equals $h_{0}(x)$. Every $f$ in $\mathscr{D}$ belongs to $\mathscr{C}_{n}$ for some $n$, so $\left|\phi_{x}(f)\right| \leqq h_{0}(x)\|f\|_{\infty}$ for every $f$ in $\mathscr{D}$. Therefore $\phi_{x}$ extends to a bounded linear functional (still denoted by $\phi_{x}$ ) on $C(Y)$ with norm $h_{0}(x)$.

For $x \in X \backslash X_{0}$, the map $\phi_{x}$ is positive since $\left\|\phi_{x}\right\|=\phi_{x}(1)$. By the Riesz Representation Theorem, there exists a positive Borel measure $\mu_{x}$ on $Y$ such that $\pi(T f)(x)=\int f(y) \mu_{x}(d y)$ for every $f$ in $\mathscr{D}$. Finally, define $\mu_{x}$ for $x \in X_{0}$, by $\mu_{x}=0$.

To show that $\left\{\mu_{x}\right\}$ is the required kernel, let $\mathscr{C}$ be the set of all $f$ in $L$ for which

$$
(T f)(x)=\int f(y) \mu_{x}(d y), \text { for } m_{1} \text {-almost every } x .
$$

We must show that $\mathscr{C}=L$. First we show that $\mathscr{C}$ contains $C(Y)$. We have already established that $\mathscr{D} \subset \mathscr{C}$. Let $f \in C(Y)$ and let $f_{n} \in \mathscr{D}$ be such that $f_{n} \rightarrow f$ uniformly. Let $\alpha_{n}=\left\|f_{n}-f\right\|_{\infty}$, so $-\alpha_{n} \leqq f_{n}-f \leqq \alpha_{n}$ and hence $-\alpha_{n} T 1 \leqq T f_{n}-T f \leqq \alpha_{n} T 1$. So $T f_{n} \rightarrow$ $T f$ almost everywhere. On the other hand, the dominated convergence theorem implies that $\int f_{n}(y) \mu_{x}(d y) \rightarrow \int f(y) \mu_{x}(d y)$. This shows that $f \in \mathscr{C}$.

The dominated convergence theorem and the order-continuity of $T$ (condition (ii)) imply that $\mathscr{C}$ is a monotone class, i.e., if $f_{n} \in \mathscr{C}$, 
$f_{n} \geqq 0, f_{n} \uparrow f$, and $f \in L$, then $f \in \mathscr{C}$. But every monotone class containing $C(Y)$ must also contain every characteristic function and hence must also contain $L$. Thus $\mathscr{C}=L$.

It remains only to show that $\mu_{x}$ can be chosen so that $\mu_{x}(B)$ is a Borel function of $x$ for every $B \in \mathscr{B}$. To prove this, let $\left\{B_{n}\right\}$ be a countable generating family for $\mathscr{B}$. Since $\mu_{x}\left(B_{n}\right)=\left(T 1_{B_{n}}\right)(x)$ almost everywhere, there is an $m_{1}$-null set $E$ such that the maps $x \rightarrow \mu_{x}\left(B_{n}\right)$ are Borel functions from $X \backslash E$ into the real numbers. Redefine $\mu_{x}$ for $x \in E$ to be 0 , so the maps $x \rightarrow \mu_{x}\left(B_{n}\right)$ become Borel functions on $X$. The measurability of $x \rightarrow \mu_{x}(B)$ for any $B \in \mathscr{B}$ follows easily.

CoROLlaRy 3.3. Let $S$ and $T$ be positive linear operators from $L$ into $M(X)$ such that $S \leqq T$. If $T$ is an integral (respectively, pseudo-integral) operator, then so is $S$.

Proof. If $T$ is a pseudo-integral operator, then $T$ is order-continuous. The operator $S$ must also be order-continuous and hence is a pseudo-integral operator.

If $\mu$ and $\nu$ are the measures inducing $T$ and $S$ respectively, then $\nu \leqq \mu$ by Lemma 3.1. If $T$ is an integral operator, then $\mu$, and hence also $\nu$, is absolutely continuous with respect to $m_{1} \times m_{2}$. Thus $S$ is an integral operator.

CoRollaRY 3.4. Every positive operator from $L^{p}(Y)$ into $L^{q}(X)$, $1 \leqq p<\infty, 1 \leqq q \leqq \infty$ is a pseudo-integral operator.

Proof. Let $T$ be a positive operator from $L^{p}(Y)$ into $L^{q}(X)$ and let $f_{n} \in L^{p}(Y), f_{n} \downarrow 0$. By the monotone convergence theorem, $\left\|f_{n}\right\|_{p} \rightarrow 0$. Since $T$ is automatically norm bounded [15, p. 84], we also have $\left\|T f_{n}\right\|_{q} \rightarrow 0$. Since $\left\{T f_{n}\right\}$ is a decreasing sequence, we must have $T f_{n} \rightarrow 0$ almost everywhere. Thus $T$ is order-continuous, and hence is a pseudo-integral operator.

The following result is stated in [10], but the proof given there seems to be incomplete.

CoROLLARY 3.5. Every bounded operator from $L^{1}(Y)$ into $L^{1}(X)$ is a pseudo-integral operator.

Compare also [9, Corollary VI. 8.9] where $X$, rather than $Y$, is assumed to be a compact metric space.

Proof. For a bounded operator $T$ from $L^{1}(Y)$ into $L^{1}(X)$, it is well-known that there are positive bounded operators $T_{1}$ and $T_{2}$ such that $T=T_{1}-T_{2}$ (in the complex case, $T=T_{1}-T_{2}+i T_{3}-i T_{4}$, 
where every $T_{j}$ is a positive bounded operator), see [7]. The result follows from the preceding corollary.

4. Order properties. For terminology and notation concerning vector lattices and operators between them we refer to Schaefer [15]. When the vector lattices under consideration are lattices of equivalence classes of measurable functions, the notions of order convergence and order continuity can be reformulated in terms of the more familiar concepts of measure theory. In particular, in a vector lattice $L$ of equivalence classes of measurable functions, a sequence of functions $\left\{f_{n}\right\}$ order-converges to 0 if and only if it converges to zero almost everywhere and dominatedly, i.e., $\left|f_{n}\right| \leqq f \in L$ and $f_{n} \rightarrow 0$ (a.e.). In the lattice $M(X)$, the condition of domination is automatically satisfied in the presence of almost everywhere convergence; see [15], p. 141, exercise 2(c).

Let $T: L \rightarrow L^{\prime}$ be a linear operator between vector lattices $L$ and $L^{\prime}$ of equivalence classes of measurable functions. Then $T$ is ordercontinuous if and only if $0 \leqq f_{n} \leqq f \in L, f_{n} \rightarrow 0$ a.e. implies that $T f_{n} \rightarrow 0$ a.e. and $\left|T f_{n}\right| \leqq g \in L^{\prime}$. Again if $L^{\prime}=M(X)$, the condition that $\left|T f_{n}\right| \leqq g$ is redundant. Order-continuous operators are called (0)-linear operators in [18], p. 214.

THEOREM 4.1. Let $T$ be a pseudo-integral operator from $L$ into $M(X)$ with kernel $\mu$. Then $T$ is order-bounded (regular) and the operators $T^{+}, T^{-}$, and $|T|$ are pseudo-integral operators with kernels, $\mu^{+}, \mu^{-}$, and $|\mu|$ respectively.

Proof. The measure $|\mu|$ induces a positive operator $S$ from $L$ into $M(X)$. Since $-|\mu| \leqq \mu \leqq|\mu|$, we must have $-S \leqq T \leqq S$. Therefore $T$ is order-bounded and $|T| \leqq S$. By Corollary 3.3, $|T|$ is a pseudo-integral operator whose kernel $\nu$ satisfies $\nu \leqq|\mu|$. On the other hand, $0 \leqq 2 T^{-}=|T|-T=T_{\nu-\mu}$, and so $\nu-\mu$ is a positive measure. Similarly $T_{\nu+\mu}=2 T^{+}$and hence $\nu+\mu$ is a positive measure. Therefore $\nu \geqq|\mu|$. Thus we have $\nu=|\mu|$, and so $|T|=T_{|\mu|}$. It follows that $T^{+}=(T+|T|) / 2=T_{\mu^{+}}$and $T^{-}=T_{\mu^{-}}$.

COROLLARY 4.2 [12]. Let $T$ be an integral operator with kernel $k$. Then $T^{+}, T^{-}$, and $|T|$ are integral operators with kernels $k^{+}$, $k^{-}$, and $|k|$ respectively.

Proof. Use Theorem 4.1 and Proposition 2.6.

CoROLLARY 4.3. Let $T_{\mu}$ and $T_{\nu}$ be pseudo-integral operators from $L$ into $M(X) . \quad$ Then $\sup \left(T_{\mu}, T_{\nu}\right)=T_{\mu \vee \nu}$ and $\inf \left(T_{\mu}, T_{\nu}\right)=T_{\mu^{\prime} \nu}$. 
REMARK. $\mu \vee \nu$ and $\mu \wedge \nu$ are the usual supremum and infimum of $\mu$ and $\nu$ in the lattice of local measures, i.e., $\mu \vee \nu=\nu+(\mu-\nu)^{+}$, and $\mu \wedge \nu=\nu-(\mu-\nu)^{-}$.

Theorem 4.1 establishes that the set of pseudo-integral operators is an order-ideal in the vector lattice $\mathscr{L}_{0}(L, M(X))$ of all orderbounded (regular) operators from $L$ into $M(X)$. We will show later (Corollary 5.3) that the pseudo-integral operators form a band (or a component in the terminology of [18]) in $\mathscr{L}_{0}(L, M(X))$. Recall that a band (a component) in a vector lattice $\mathscr{L}$ is by definition an order-ideal $\mathscr{I}$ with the property that whenever $x_{\alpha} \in \mathscr{J}$ and $x=$ $\sup \left\{x_{\alpha}\right\}$ exists in $\mathscr{L}$, we must have $x \in \mathscr{F}$. (It would be more descriptive to call an ideal with this property an order-closed ideal.)

\section{The characterization theorem.}

LEMMA 5.1. Let $T$ be an order-continuous operator from $L$ into $M(X)$. Then $T$ is order-bounded, and the operators $T^{+}, T^{-}$, and $|T|$ are order-continuous.

Proof. The proof is given in [18, p. 214 and p. 216] for operators between more general vector lattices. We give a sketch of the proof.

To prove that $T$ is order-bounded it is enough to consider sequences [18, p. 154], i.e., if $\left|f_{n}\right| \leqq f \in L$, we must show that $\sup \left\{T f_{n}\right\}$ exists. To prove this, it suffices to show that $\lambda_{n} T f_{n} \rightarrow 0$ a.e. whenever $\left\{\lambda_{n}\right\}$ is a sequence of positive real numbers converging to 0 . But this follows from the order-continuity of $T$.

To prove that $T^{+}$is order-continuous, let $f_{n} \geqq 0, f_{n} \uparrow f \in L$. We must show that $T^{+} f=\sup \left\{T^{+} f_{n}\right\}$. If $0 \leqq g \leqq f$, then $\left(g \wedge f_{n}\right) \uparrow g$ and hence $T g=\sup T\left(g \wedge f_{n}\right)=\sup T^{+}\left(g \wedge f_{n}\right) \leqq \sup T^{+} f_{n}$. So $T^{+} f=\sup \{T g$ : $0 \leqq g \leqq f\} \leqq \sup T^{+} f_{n}$. The reverse inequality is trivial. This shows that $T^{+}$is order-continuous and so $T^{-}$and $|T|$ must also be ordercontinuous.

THEOREM 5.2. Let $T$ be a linear operator from $L$ into $M(X)$. The following conditions are equivalent.

(i) $T$ is a pseudo-integral operator.

(ii) $T$ is order-continuous, i.e., if $0 \leqq f_{n} \leqq f \in L$ and $f_{n} \rightarrow 0$ a.e., then $T f_{n} \rightarrow 0$ a.e.

Proof. If $T$ is a pseudo-integral operator, then $T^{+}$and $T^{-}$are pseudo-integral operators by Theorem 4.1. It follows from Theorem 3.2 that $T^{+}$and $T^{-}$are order-continuous, and hence $T=T^{+}-T^{-}$ 
is order-continuous.

Conversely, if $T$ is order-continuous, apply Lemma 5.1 and Theorem 3.2 to conclude that $T$ is the difference between two pseudointegral operators, and so $T$ itself must be a pseudo-integral operator.

COROLlaRY 5.3. The pseudo-integral operators form a band (an order-closed ideal) in the vector lattice of order-bounded operators from $L$ into $M(X)$.

Proof. It is known [18, p. 216], that the order-continuous operators between two vector lattices from a band in the lattice of regular operators.

We are now in a position to show that the measurability condition 1.1 (ii) is redundant as far as operators are concerned.

Proposition 5.4. Let $x \rightarrow \mu_{x}$ be a map of $X$ into the space of bounded Borel measures on $Y$, and let $L$ be an order-ideal of $M(Y)$ and $T$ an operator from $L$ into $M(X)$ such that

(a) every $f$ in $L$ belongs to $L^{1}\left(\left|\mu_{x}\right|\right)$ for almost every $x$,

(b) $(T f)(x)=\int f(y) \mu_{x}(d y)$ for $f \in L$.

Then there are measures $\nu_{x}$ such that

(c) $\nu_{x}=\mu_{x}$ for almost every $x$,

(d) for every $B$ in $\mathscr{B}$, the maps $x \rightarrow \nu(B)$ and $x \rightarrow\left|\nu_{x}\right|(B)$ are Borel functions.

Proof. By the dominated convergence theorem, the operator $T$ is order-continuous. Theorem 5.2 implies the existence of $\nu_{x}$.

6. More general measure spaces. In this section we generalize our results to the case of a separable (not necessarily standard) measure space $\left(Y, \mathscr{B}, m_{2}\right)$. In this case, we will use the term "pseudointegral operator" to mean an operator induced by a measure kernel $\mu$ as in Lemma 2.3. Since the theorem of disintegration of measures is not available in the present case, we may not be able to obtain an explicit representation of the operator as in (0.2). Operators given by $(0.2)$ form a subclass of what we now call pseudo-integral operators.

Examination of the proofs of our previous results shows that they extend to the present general case once Theorem 3.2 has been so extended. In what follows $\left(Y, \mathscr{B}, m_{2}\right)$ is a separable finite measure space.

LemMa 6.1. Let $T$ be a positive pseudo-integral operator from 
$L$ into $M(X)$, then $T$ is order-continuous.

Proof. Let $f_{n} \downarrow 0$. We must show that $T f_{n} \downarrow 0$. Toward this end, let $g=\inf \left\{T f_{n}\right\}$, and let $X_{j}=\left\{x:\left(T f_{1}\right)(x) \leqq j\right\}$. If suffices to show that $g=0$ a.e. on every $X_{j}$, and so we may assume that $T f_{1}$ is a bounded function. Let $\mu$ be the kernel of $T$, and $A \in \mathscr{A}$, the monotone convergence theorem shows that

$$
\begin{aligned}
\int g(x) 1_{A}(x) m_{1}(d x) & =\lim \int\left(T f_{n}\right)(x) 1_{A}(x) m_{1}(d x) \\
& =\lim \iint f_{n}(y) 1_{A}(x) \mu(d x, d y)=0 .
\end{aligned}
$$

Thus $g=0$. This proves the Lemma.

For any separable measure space $\left(Y, \mathscr{B}, m_{2}\right)$, there is a compact metric space $Y^{\prime}$, a Borel measure $m_{2}^{\prime}$ on $Y^{\prime}$ and an isomorphism of the measure algebra $\left(Y, m_{2}\right)$ onto the measure algebra of $\left(Y^{\prime}, m_{2}^{\prime}\right)$ (see [11, p. 173] for the nonatomic case). This isomorphism induces a one-to-one positive linear map \& of $M(Y)$ onto $M\left(Y^{\prime}\right)$, see [8, pp. 252-254] for details. It is easy to see that $\psi$ preserves almost everywhere convergence, that is $f_{n} \rightarrow 0$ a.e. if and only if $\psi\left(f_{n}\right) \rightarrow 0$ a.e. This follows from the observation that $\psi$ preserves the order structure and the fact that $f_{n} \rightarrow 0$ a.e. if and only if there is a decreasing sequence of positive functions $\left\{g_{n}\right\}$ such that $\left|f_{n}\right| \leqq g_{n}$ and $\inf \left\{g_{n}\right\}=0$.

For an operator $T$ from $L$ into $M(X)$, let $\hat{T}$ be the operator from $\psi(L)$ into $M(X)$ defined by $\widehat{T}(f)=T\left(\psi^{-1}(f)\right)$. It is straightforward to see that $\hat{T}$ is positive if and only if $T$ is positive and that $\hat{T}$ is order-continuous if and only if $T$ is order-continuous.

THEOREM 6.2. Let $T$ be a positive operator from $L$ into $M(X)$. The following conditions are equivalent.

(i) $T$ is a pseudo-integral operator, in the sense that it has a measure kernel on $X \times Y$.

(ii) $T$ is order-continuous.

Proof. The implication (i) $\Rightarrow$ (ii) has already been proved. Assume that $T$ is order-continuous. Therefore $\hat{T}$ is order-continuous and so by Theorem 3.2 it is a pseudo-integral operator induced by a measure $\hat{\mu}$ on $X \times Y^{\prime}$. Define $\mu$ on the measurable rectangles of $X \times Y$ by $\mu(A \times B)=\int 1_{A}(x)\left(T 1_{B}\right)(x) m_{1}(d x)$. Thus $\mu(A \times B)=\hat{\mu}(A \times \psi(B))$. In order to show that $\mu$ extends to a countably additive measure on $\mathscr{A} \otimes \mathscr{B}$ it suffices to show that $\mu$ is countably additive on rectangles $[14$, p. 224]. But this is satisfied because $\hat{\mu}$ is countably additive. 
In order to prove that $T$ is a pseudo-integral operator induced by the measure $\mu$, we must prove the following:

(a) For every $f$ in $L$, the ideal of sets $\mathscr{A}_{f}=\left\{A: A \in \mathscr{A}, 1_{A}(x) f(y) \in\right.$ $\left.L^{1}(\mu)\right\}$ generates as a $\sigma$-algebra.

$A \in \mathscr{A}_{f}$.

(b) $\int(T f)(x) 1_{A}(x) m_{1}(d x)=\iint f(y) g(x) \mu(d x, d y)$ for every $f \in L$ and

When $f$ is a simple function, each of the conditions (a) and (b) is obviously satisfied in view of the definition of $\mu$. Their validity for arbitrary $f$ in $L$ follows from the order-continuity of $T$ and the monotone convergence theorem.

\section{REFERENCES}

1. M. B. Abrahamse and T.L. Kriete, The spectral multiplicity of a multiplication operator, Indiana Univ. Math. J., 22 (1973), 845-857.

2. W. Arveson, Operator algebras and invariant subspace, Ann. of Math., (2), 100 (1974), 433-532.

3. — An Invitation to $C^{*}$-Algebras, Springer-Verlag, New York, 1976.

4. R. B. Ash, Real Analysis and Probability, Academic Press, New York, 1972.

5. N. Bourbaki, Eléménts de Mathématique, Livre VI: Intégration. Chapitre 6: Intégration vectorielle, Hermann, Paris, 1959.

6. A. V. Bukhvalov, Integral representation of linear operators, Zap. Naucn, Sem. Leningrad. Otdel. Mat. Inst. Steklov. (LOMI), 47 (1974), 5-14.

7. R. V. Chacon and U. Krengel, Linear modulus of a linear operator, Proc. Amer. Math. Soc., 15 (1964), 553-559.

8. J. L. Doob, Stochastic Processes, Wiley, New York, 1953.

9. N. Dunford and J. T. Schwartz, Linear Operators, Part I, Interscience, New York, 1958.

10. H. Fakhoury, Représentations d'opérateurs à valeurs dans $L^{1}(X, \Sigma, \mu)$, Math. Ann., 240 (1979), 203-212.

11. P. R. Halmos, Measure Theory, Van Nostrand, New York, 1950.

12. W. A. J. Luxemburg and A.C. Zaanen, The linear modulus of an order bounded linear transformation, Indag. Math., 33 (1971), 422-447.

13. G. Mackey, Borel structures in groups and their duals, Trans. Amer. Math. Soc., 85 (1957), 265-311.

14. H. L. Royden, Real Analysis, Macmillan, New York, 1963.

15. H.H. Schaefer, Banach Lattices and Positive Operators, Springer-Verlag, New York, 1974.

16. A. R. Schep, Kernel operators, Indag. Math., 41 (1979), 39-53.

17. A. R. Sourour, Pseudo-integral operators, Trans. Amer. Math. Soc., 253 (1979), 339-363.

18. B.Z. Vulikh, Introduction to the Theory of Partially Ordered Spaces, WoltersNoordhoff, Groningen, 1967.

Received March 3, 1980 and in revised form July 30, 1980.

UNIVERSITY OF VICTORIA

VICTORIA, BRITISH COLUMBIA

CANADA V8W $2 Y 2$ 


\section{PACIFIC JOURNAL OF MATHEMATICS}

\section{EDITORS}

DONALD BABBITT (Managing Editor)

University of California

Los Angeles, California 90024

\section{Hugo Rossi}

University of Utah

Salt Lake City, UT 84112

C. C. MOore and Arthur AGuS

University of California

Berkeley, CA 94720
J. DugundJI

Department of Mathematics University of Southern California Los Angeles, California 90007

R. FinN and J. Milgram Stanford University Stanford, California 94305

ASSOCIATE EDITORS
R. ARENS
E. F. BECKENBACH
B. H. NeumanN
F. WOLF
K. YOSHIDA

\section{SUPPORTING INSTITUTIONS}

UNIVERSITY OF ARIZONA

UNIVERSITY OF BRITISH COLUMBIA

CALIFORNIA INSTITUTE OF TECHNOLOGY

UNIVERSITY OF CALIFORNIA

MONTANA STATE UNIVERSITY

UNIVERSITY OF NEVADA, RENO

NEW MEXICO STATE UNIVERSITY

OREGON STATE UNIVERSITY
UNIVERSITY OF OREGON

UNIVERSITY OF SOUTHERN CALIFORNIA

STANFORD UNIVERSITY

UNIVERSITY OF HAWAII

UNIVERSITY OF TOKYO

UNIVERSITY OF UTAH

WASHINGTON STATE UNIVERSITY

UNIVERSITY OF WASHINGTON 


\section{Pacific Journal of Mathematics}

Vol. 99, No. $1 \quad$ May, 1982

Mariano Giaquinta, Jindrich Necas, O. John and J. Stará, On the

regularity up to the boundary for second order nonlinear elliptic systems . . 1

Siegfried Graf, Realizing automorphisms of quotients of product $\sigma$-fields . . 19

Alfred Washington Hales and Ernst Gabor Straus, Projective colorings . . . 31

Sandra Hayes, The weak Nullstellensatz for finite-dimensional complex

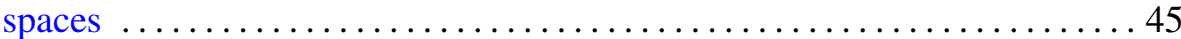

Gerald Norman Hile and Murray Harold Protter, The Cauchy problem

and asymptotic decay for solutions of differential inequalities in Hilbert

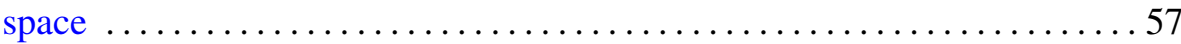

Robert D. Little, Projective space as a branched covering with orientable

branch set ......................................... 89

Jaroslav Mach, On the proximinality of Stone-Weierstrass subspaces . . . . . 997

John C. Morgan, II, On product bases ...................... 105

K. Balakrishna Reddy and P. V. Subrahmanyam, Altman's contractors

and fixed points of multivalued mappings . .................. 127

James Ted Rogers Jr., Decompositions of homogeneous continua . . . . . . . 137

Ahmed Ramzy Sourour, Characterization and order properties of

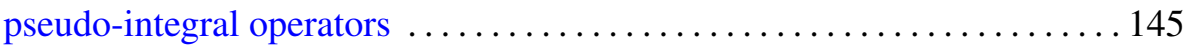

Robert Moffatt Stephenson Jr., Pseudocompact and Stone-Weierstrass

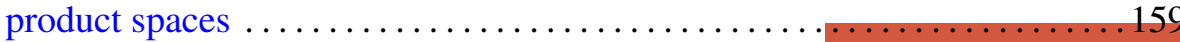

Bruce Stewart Trace, On attaching 3-handles to a 1-connected

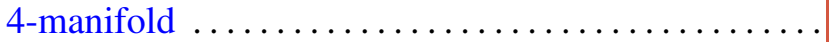

Akihito Uchiyama, The construction of certain BMO functions and the corona problem

Thomas Alva Whitehurst, An application of orthogonal polynomials to random walks ..............................

David J. Winter, Root locologies and idempotents of Lie and nonassociative algebras

William Robin Zame, The classification of uniform algebras on plane domains 